\title{
Original Management of incidentally detected gallbladder Alicle carcinomas in a high prevalence area of gall bladder cancer
}

\author{
Kallianpur AA, ${ }^{1}$ Gupta N, ${ }^{2}$ Vinod N, ${ }^{2}$ Rakesh G, ${ }^{2}$ Samra SS, ${ }^{3}$ \\ Goyal $S^{4}$
}

ABSTRACT

Departments of Surgical Oncology, ${ }^{1}$ Radiation Oncology, ${ }^{2}$ and Surgery, ${ }^{3}$ Grecian Cancer and Superspecialty Hospital, Mohali,

Department of Surgery, ${ }^{4}$

Government Medical College and

Hospital,

Sector-32, Chandigarh,

Punjab, India

Correspondence:

Dr. Nidhi Gupta

Email:nidhiprinja@gmail.com
Background: There is an increasing incidence of advanced unresectable gallbladder cancer even in patients who undergo re-exploration and these cases are marked by poor survival even after undergoing curative resection and adjuvant chemotherapy. Lack of suspicion during primary surgery, unavailability of frozen section facilities and delayed referrals are believed to contribute to this high incidence.

Aim: Our aim was to evaluate the results of re-surgery in incidental gallbladder cancers detected after open or laparoscopic cholecystectomy and to assess the outcome in patients who underwent complete radical cholecystectomy and adjuvant therapy.

Method: We retrospectively analyzed the data from a prospectively maintained computerized database of all patients with incidentally detected gallbladder cancers operated in the Department of Surgical Oncology, from June 2006 to January 2013.

Results: Forty-two patients with incidental gallbladder cancer were re-explored. The median time of re-exploration after initial surgery was 65 days. Eighteen (43\%) patients were found inoperable due to locally advanced unresectable or metastatic disease. Among the 24 (57\%) patients who underwent completion radical cholecystectomy, 11 developed recurrence over a median time of 11 months.

Conclusion: Despite the dismal prognosis, more than half of the incidentally detected gallbladder carcinoma patients could receive curative treatment. Identification of patients with incidentally discovered gallbladder cancer and early referral to an oncology center may ensure these patients receive curative resection thereby increasing their chances for longterm disease free survival.

KEYWORDS: Cholecystectomy, incidental, gallbladder carcinoma; re-surgery

\section{Introduction}

Gallbladder cancer is the most frequently encountered malignancy of the biliary system. There is a wide variation in reported epidemiology and burden of the disease. Western studies report an annual incidence of 1-2 gallbladder cancer cases per 100,000 population. ${ }^{1}$ In India gallbladder cancer is one of the most common gastrointestinal malignancies with the highest incidence reported in the northern parts of the country, especially along the Gangetic plains. ${ }^{2}$ Delhi, the capital of the country, reports an age adjusted incidence of gallbladder cancer of 10.6 patients per 100,000 population, which is incidentally the world's highest rate for women suffering from this cancer. ${ }^{3}$ 
Incidentally detected gallbladder cancer is an uncommon entity and is frequently discovered in cholecystectomy specimens operated for benign gallstones. Such incidentally discovered cancer has best prognosis if treated at the earliest with radical surgery and adjuvant therapy. Any delay in referral diminishes the chances of a curative resection. The objective of the present study was to assess the management and results of such incidental gallbladder cancer patients being referred to our Department of Surgical Oncology.

\section{Methods}

Relevant clinical and surgical data was retrieved from a computerized database of gallbladder cancer patients treated at our Department of Surgical Oncology. Patients who were diagnosed with incidental gallbladder cancer between June 2006 and January 2013 were included for this retrospective analysis. A case of incidentally discovered gallbladder cancer was defined as a patient who underwent simple cholecystectomy and was discovered to harbor gallbladder cancer on histopathology examination of operative specimen(s). Untreated patients with radiological findings of gallbladder cancer were excluded. All patients with incidentally detected gallbladder cancer, underwent computerized tomography (CT) of abdomen to determine the extent of residual or recurrent disease and chest X-ray to rule out metastasis. Only those patients were chosen for re-surgery who were deemed operable radiologically.

All patients underwent exploratory laparotomy and were assessed for resectability based on local advancement or metastasis of the cancer. The surgical approach for resectable incidental gallbladder cancer has been consistent at our centre and includes wide excision of the scar, portal lymphadenectomy and hepatic resection with $2 \mathrm{~cm}$ margin of the gallbladder bed. Adjuvant postoperative treatment is given to patients with $\mathrm{T} 2$ or worse disease and pathological node positivity. All patients were followed three monthly for first two years and six monthly for next three years and annually thereafter. Patients were assessed clinically and with abdominal CT scan for evidence of recurrence. All loco-regional relapses and systemic metastasis were documented. A descriptive analysis of demography and clinico-pathological features, surgical treatment, adjuvant treatment offered and long-term outcome was carried out. Tumor staging was reported according to the American Joint Committee on Cancer (7th edition) TNM staging guidelines.

\section{Results}

Of the 78 patients with gallbladder cancers referred for surgery, $42(53.8 \%)$ were incidentally detected to harbor gallbladder cancer following cholecystectomy and subsequent histopathology evaluation of the specimen. These 42 patients comprised of 36 women and six men, with overall median age of 46 years (range: 25-69 years). All 42 patients had undergone simple cholecystectomy in a general hospital for benign gallbladder disease. The preoperative diagnosis of patients before undergoing simple cholecystectomy is shown in Table 1. Forty-three percent (18 of 42) patients had undergone laparoscopic and fifty-seven percent (24 of 42) an open cholecystectomy for their benign gallbladder disease (Table 2). Histopathology examination of the cholecystectomy specimen revealed adenocarcinoma in all patients.

While 35 patients were asymptomatic after cholecystectomy, three complained of pain and four developed portal metastasis. The median duration among patients who underwent initial simple cholecystectomy and re-surgery after

Table 1: Preoperative sonographic diagnosis prior to cholecystectomy, in patients later found to have incidental gallbladder cancer

\begin{tabular}{lc}
\hline Preoperative Diagnosis & Number of patients \\
\hline Acalculus cholecystitis & 1 \\
Acute cholecystitis with cholelithiasis & 1 \\
Chronic cholecystitis with cholelithiasis & 7 \\
Cholelithiasis with asymmetric gall bladder & 5 \\
wall thickening & \\
Cholelithiasis & 28 \\
\hline
\end{tabular}

Table 2: Summary of inoperable, operable and recurrence cases of incidental gallbladder cancer

\begin{tabular}{|c|c|c|c|c|c|}
\hline & $\begin{array}{l}\text { Inoperable } \\
\text { cancer }\end{array}$ & incidental & gallbladder & $\begin{array}{l}\text { Operable incidental } \\
\text { gallbladder cancer }\end{array}$ & $\begin{array}{l}\text { Recurrence after } \\
\text { completion radical } \\
\text { cholecystectomy }\end{array}$ \\
\hline $\begin{array}{l}\text { Type of cholecystectomy } \\
\text { (n) }\end{array}$ & $\begin{array}{l}\text { Number of } \\
\text { patients }(\%)\end{array}$ & $\begin{array}{l}\text { Local } \\
\text { cause }\end{array}$ & $\begin{array}{l}\text { Systemic } \\
\text { cause }\end{array}$ & $\begin{array}{l}\text { Number of patients } \\
(\%)\end{array}$ & $\begin{array}{l}\text { Number of patients } \\
(\%)\end{array}$ \\
\hline Open cholecystectomy (24) & $11(45.8 \%)$ & 6 & 5 & $13(54.2 \%)$ & $7(53.8 \%)$ \\
\hline $\begin{array}{l}\text { Laparoscopic chole- } \\
\text { cystectomy }(18)\end{array}$ & $7(38.9 \%)$ & 4 & 3 & $11(61.1 \%)$ & $4(36.3 \%)$ \\
\hline
\end{tabular}


discovery of incidental gallbladder cancer was 65 days (range: 19 - 145days). Only six patients could be re-operated within 45 days of initial simple cholecystectomy. Twenty four (57.1\%) patients underwent complete radical cholecystectomy and the remaining eighteen $(42.9 \%)$ were re-explored only to find unresectable or metastatic disease. Ten patients were inoperable because of metastatic disease and remaining eight had unresectable locally advanced disease (four patients with duodenal and four with porta involvement). Postoperative complication was seen in six patients, with four contracting wound infection and two postoperative ileus, all of which was managed conservatively.

Of the patients who received curative surgery $13(54.2 \%)$ had stage III, and $11(45.8 \%)$ stage II gallbladder carcinoma. Twenty one patients received concomitant 5FU and leucovorin during the first and last week of radiotherapy up to a dose of 45 Grays delivered over five weeks using 3D conformal radiotherapy technique (Macdonald's chemoradiotherapy regimen). ${ }^{4,5}$ Two patients received gemcitabine and carboplatin based chemotherapy and one patient received 50 Grays adjuvant radiotherapy only. Among the patients who underwent complete radical cholecystectomy, eleven (45.83\%) developed recurrence after a median time of 11 months (range: 2.6-30 months). Nine (69\%) and two (18\%) cases of recurrence were seen in patients with stage III and II disease, respectively. The mean survival of patients with stage II and III cancer were 74.07 and 24.34 months ( $p=0.008$ ), respectively. Recurrence was noted within 12.2 to 21.2 months after re-surgery among stage II cancer patients. The median time to recurrence of in stage III patients was 11.5 months. The two and three year survival of stage II patients was $82 \%$...... whereas that for stage III patients was $52 \%$ and $20 \%$, respectively There was no mortality in patients who underwent completion radical cholecystectomy within 45 days. The two and three year survival of patients who presented for re-surgery after 45 days of initial surgery was $35 \%$ and $29 \%$, respectively). Thirteen $(54 \%)$ patients were alive and disease free at a median followup of 14 months (range: 8-86 months).

\section{Discussion}

Incidental gallbladder cancer is defined as a tumor identified in the postoperative gallbladder specimen, typically removed for symptoms related to gallstones and not suspected for malignancy during preoperative work up or cholecystectomy.

De Stoll in 1771 was the first to report on gallbladder carcinoma. ${ }^{6}$ It is the fifth most common gastrointestinal malignancy and is often discovered when the disease is far advanced and unresectable. Gallbladder cancer is found incidentally in at least $0.3 \%$ and up to $2.9 \%$ (in high prevalence areas) of gallbladders removed for presumed benign disease. ${ }^{7-}$ ${ }^{14}$ Francisco et al in a study of cholecystectomy done for benign disease at a regional hospital in Spain, found six of 372 patients with gallbladder cancer. ${ }^{15}$

A cohort of gallbladder cancer patients who underwent surgery at three centers [Instituto Oncologico Fundacion Arturo Lopez Perez (FALP), Chile; Yokohama City University (YCU), Japan; and Memorial Sloan-Kettering Cancer Center (MSKCC), USA] between 1999 and 2007 were examined for incidental gallbladder cancer. It was detected in 152 (58.2\%) out of 261 patients who underwent cholecystectomy. The proportional differences of patients diagnosed with gallbladder after cholecystectomy among the centers were 55 (64.7\%), 9 (19.5\%) and $88(67.7 \%)$ at FALP, YCU and MSKCC, respectively. ${ }^{16}$ The gallbladder tumor was not detected on preoperative radiology in 53/107 (50\%) patients admitted to the Johns Hopkins Hospital with gallbladder cancer between February 1995 and June 2004. ${ }^{17}$ Tantia et al from India reported an incidence of $0.59 \%$ incidentally detected gallbladder cancer on histopathology of laparosopic cholecystectomy specimens. ${ }^{18}$ In the current series forty-two patients were referred to our institute after being diagnosed with gallbladder carcinoma histopathologically following cholecystectomy elsewhere.

Ultrasonography is usually the initial radiographic study obtained, when benign gallbladder disease is suspected. Gallbladder cancer may have three distinct appearances on ultrasound: (1) a mass replacing the gallbladder or invading the gallbladder bed, (2) an intraluminal gallbladder growth or polyp, or (3) asymmetric gallbladder wall thickening. These typical findings are not seen in early gallbladder cancers especially in flat or sessile cancerous polyps. ${ }^{19}$ In a series of 71 patients with early gallbladder cancer, ultrasound had a sensitivity of $53 \%$ for sessile tumors. ${ }^{20}$

Gallbladder cancers at times can be detected at the time of laparoscopy. Others are picked up after the specimen is removed and the pathology review comes out to be gallbladder carcinoma. At times when doubt arises, it is better to perform frozen section histopathology if facilities are available. Certain features which mandate further examination after frozen section include: macroscopically contracted or sclerotic mucosa, thickening of one part of gallbladder wall, normal tissue replaced 
by connective tissue in all wall layers, macroscopic mucosal color change, existence of polypoid lesions. ${ }^{10}$ Frozen section may be an inadequate and a limited diagnostic sample as the entire lesion cannot be examined. However when a surgeon intraoperatively shows a doubtful lesion to the pathologist, frozen section can be useful in making decisions in such cases, with a reported accuracy for gallbladder cancer of $88.2 \% .^{7}$ Unfortunately, it is not always possible to determine the exact depth of invasion which is critical for planning further surgical intervention. ${ }^{11}$ Patients prepared for laparoscopic or open cholecystectomy for benign disease would neither have been appropriately staged nor would have consented. Furthermore, most patients are operated at centers ill-equipped for oncological resections, which may explain the large number of referrals for completion radical cholecystectomy. Frozen section does however allow the clinician to diagnose patients with gallbladder carcinoma at an early stage and refer them to oncology centers for appropriate line of treatment. Due to unavailability of frozen section facilities in many hospitals and delay in referral, majority of patients with gallbladder cancer reach a higher centre when their disease has become inoperable. Nearly half the patients in our cohort, irrespective of whether the patients underwent laparoscopic or open cholecystectomy, were inoperable by the time they were referred to us.

The German registry founded in 1997 for prospective surveillance of all incidental gallbladder cancers in Germany, stressed early re-resection to achieve maximum disease free survival. ${ }^{21}$ The registry defined early re-resection (ERR) if performed within 45 days of undergoing simple cholecystectomy. Among the incidental gallbladder cancer patients, 200 and 85 were staged T2 and T3, respectively. The median time for re-resection after cholecystectomy was 14.4 days. Eighty-five T2 gallbladder cancer patients received ERR treatment which improved their survival from $35 \%$ to $55 \%$. This ERR benefit was not seen in patients with T3 gallbladder cancer. ${ }^{21}$ In a subsequent review 231 of 624 incidental gallbladder cancer patients received completion radical cholecystectomy within 45 days of simple cholecystectomy. All patients who received ERR could complete oncological resection either with portal lymphadenectomy with wedge or segment IV/V liver resection or extended resection that included right hepatectomy or Whipple resections. ${ }^{22}$ Shukla et al reported that $71 \%$ of patients (54/76) could undergo resurgery after detection of incidental gallbladder cancer at a median time interval of two months from initial surgery. ${ }^{23}$ The median time of referral in the present study was 65 days which explains the high incidence of inoperability seen among our patients. Metastasis was seen in $23.8 \%$ patients at reexploration in our series. Hence an early referral is imperative for taking up curative resection and achieving maximum benefit. In cases where gallbladder cancer is diagnosed at the time of primary surgery taken up for presumed benign disease, if the surgeon is unprepared for performing a hepatic resection, then the patient is best served by transfer to a more experienced center.

Surgical resection is the lone, potentially curative option for any patient with incidental gallbladder cancer and multiple studies have documented very high cure rates for patients with early stage disease. Several recent studies have reported long-term survivors after margin negative resection of stages III gallbladder tumors. ${ }^{24,25}$ Half of the patients in the series who had undergo re-resection of incidental gallbladder tumor developed loco-regional or distant recurrences. Toyonaga et al reported, $37 \%$ and $100 \%$ of patients with T2 and T3 incidental gallbladder cancer with recurrence after completion radical surgery. ${ }^{26}$

Most patients of early staged gallbladder cancer (stages $\mathrm{T} 1, \mathrm{~T} 2$ ) are discovered incidentally after cholecystectomy for benign disease. The rarity of early staged gallbladder cancer coupled with the high prevalence of benign gallbladder disease means that most patients undergo initial procedures that violate tumor planes, complicating future attempts for oncological resection. Lack of frozen section facilities in most hospitals, late referral of patients and limited tertiary care centers, result in late diagnosis of most patients by which time they have advanced stage disease. Despite aggressive tumor biology and majority of the patients being diagnosed at an advanced stage due to late referrals, every effort must be taken to ensure oncological clearance of the disease if feasible. The current study affirms that shorter time interval between primary surgery and revision radical re-surgery for incidental gallbladder cancer does seem to affect operability and thereby patient survival.

\section{References}

1. Fong Y, Malhotra S. Gallbladder cancer: recent advances and current guidelines for surgical therapy. Adv Surg. 2001;35:1-20.

2. Batra Y, Pal S, Dutta U, Desai P, Garg PK, Makharia G, et al. Gallbladder cancer in India: a dismal picture. J Gastroenterol Hepatol. 2005;20:309-14.

3. Mudur G. India has some of the highest cancer rates in the world. BMJ. 2005;330:215. 
4. Macdonald JS, Smalley SR, Benedetti J, Hundahl SA, Estes NC, Stemmermann GN, et al. Chemoradiotherapy after surgery compared with surgery alone for adenocarcinoma of the stomach or gastroesophageal junction. N Engl J Med. 2001;345:725-30.

5. Macdonald OK, Crane CH. Palliative and postoperative radiotherapy in biliary tract cancer. Surg Oncol Clin N Am. 2002;11:941-54.

6. Weinstein D, Herbert M, Bendet N, Sandbank J, Halevy A. Incidental finding of gallbladder carcinoma. Isr Med Assoc J. 2002;4:334-6.

7. Sun CD, Zhang BY, Wu LQ, Lee WJ. Laparoscopic cholecystectomy for treatment of unexpected early-stage gallbladder cancer. J Surg Oncol. 2005;91:253-7.

8. Darabos N, Stare R. Gallbladder cancer: laparoscopic and classic cholecystectomy. Surg Endosc. 2004;18:144-7.

9. Akatsu T, Ueda M, Shimazu M, Wakabayashi G, Aiura K, Tanabe $\mathrm{M}$, et al. Long-term survival of patients with gallbladder cancer detected during or after laparoscopic cholecystectomy. World $J$ Surg. 2005;29:1106-9, discussion 10.

10. Akyurek N, Irkorucu O, Salman B, Erdem O, Sare M, Tatlicioglu E. Unexpected gallbladder cancer during laparoscopic cholecystectomy. $J$ Hepatobiliary Pancreat Surg. 2004;11:357-61.

11. Kwon AH, Imamura A, Kitade H, Kamiyama Y. Unsuspected gallbladder cancer diagnosed during or after laparoscopic cholecystectomy. J Surg Oncol. 2008;97:241-5.

12. Yamamoto H, Hayakawa N, Kitagawa Y, Katohno Y, Sasaya T, Takara D, et al. Unsuspected gallbladder carcinoma after laparoscopic cholecystectomy. J Hepatobiliary Pancreat Surg. 2005;12:391-8..

13. Yeh CN, Jan YY, Chen MF. Management of unsuspected gallbladder carcinoma discovered during or following laparoscopic cholecystectomy. Am Surg. 2004;70:256-8.

14. Giuliante F, Ardito F, Vellone M, Clemente G, Nuzzo G. Portsites excision for gallbladder cancer incidentally found after laparoscopic cholecystectomy. Am J Surg. 2006;191:114-6.

15. Morera Ocon FJ, Ballestin Vicente J, Ripoll Orts F, Landete Molina F, Garcia-Granero Ximenez M, Millan Tarin J, et al.
[Gallbladder cancer in a regional hospital]. Cir Esp. 2009;86:219-23.

16. Butte JM, Matsuo K, Gonen M, D’Angelica MI, Waugh E, Allen PJ, et al. Gallbladder cancer: differences in presentation, surgical treatment, and survival in patients treated at centers in three countries. J Am Coll Surg. 2011;212:50-61.

17. Shih SP, Schulick RD, Cameron JL, Lillemoe KD, Pitt HA, Choti MA, et al. Gallbladder cancer: the role of laparoscopy and radical resection. Ann Surg. 2007;245:893-901.

18. Tantia O, Jain M, Khanna S, Sen B. Incidental carcinoma gall bladder during laparoscopic cholecystectomy for symptomatic gall stone disease. Surg Endosc. 2009;23:2041-6.

19. Onoyama H, Yamamoto M, Takada M, Urakawa T, Ajiki T, Yamada I, et al. Diagnostic imaging of early gallbladder cancer: retrospective study of 53 cases. World J Surg. 1999;23:708-12.

20. Tsuchiya Y. Early carcinoma of the gallbladder: macroscopic features and US findings. Radiology. 1991;179:171-5.

21. Goetze TO, Paolucci V. Immediate re-resection of T1 incidental gallbladder carcinomas: a survival analysis of the German Registry. Surg Endosc. 2008;22:2462-5.

22. Goetze TO, Paolucci V. Adequate extent in radical re-resection of incidental gallbladder carcinoma: analysis of the German Registry. Surg Endosc. 2010;24:2156-64.

23. Shukla PJ, Barreto G, Kakade A, Shrikhande SV. Revision surgery for incidental gallbladder cancer: factors influencing operability and further evidence for T1b tumours. HPB (Oxford). 2008;10:43-7.

24. Dixon E, Vollmer CM, Jr., Sahajpal A, Cattral M, Grant D, Doig $\mathrm{C}$, et al. An aggressive surgical approach leads to improved survival in patients with gallbladder cancer: a 12-year study at a North American Center. Ann Surg. 2005;241:385-94.

25. Kondo S, Nimura Y, Hayakawa N, Kamiya J, Nagino M, Uesaka K. Extensive surgery for carcinoma of the gallbladder. Br J Surg. 2002;89:179-84.

26. Toyonaga T, Chijiiwa K, Nakano K, Noshiro H, Yamaguchi K, Sada $\mathrm{M}$, et al. Completion radical surgery after cholecystectomy for accidentally undiagnosed gallbladder carcinoma. World J Surg. 2003;27:266-71. 